Hamburg

\title{
Aus dem Programm: Kultursensibel pflegen
}

_ Am 4. und 5. November 2016 findet im Radisson Blu Hotel in Hamburg der 14. Gesundheitspflege-Kongress von Springer Pflege statt. Erwartet werden wieder rund 800 Kongressteilnehmer.

Was müssen Pflegekräfte wissen, die Menschen mit Migrationshintergrund versorgen? Die Hochschule für Angewandte Wissenschaften (HAW) Hamburg hat ein Schulungsprogramm für professionell Pflegende entwickelt, um kultursensible Kompetenzen zu vermitteln. „Pflegekräfte sind zwar sehr aufgeschlossen anderen Kulturen gegenüber, haben aber oft auch noch Berührungsängste", berichtet Prof. Dr. Corinna PetersenEwert, eine der Leiterinnen des HAW-Projektes, das auf dem Kongress vorgestellt wird.
Das Projekt konzentriert sich auf die Versorgung Pflegebedürftiger mit türkischen und polnischen Wurzeln. Mit 18 bzw. 13\% der Gesamtbevölkerung sind das die beiden größten Gruppen mit Migrationshintergrund in Hamburg. Tee trinken statt Small Talk - in der türkischen Kultur funktioniert die Kontaktaufnahme anders als in der deutschen. "Gleichgeschlechtliche Pflege, andere Ernährungsgewohnheiten und kulturelle Bräuche - es gibt einige Besonderheiten zu beachten", so Petersen-Ewert. Themen wie Patientenorientierung, Pflege- und Ergebnisqualität, Auswirkungen des Pflegeberufsgesetzes, Arbeitszeitgestaltung stehen ebenso auf dem Programm wie Schulung von Patienten und Angehörigen sowie neue Lernformen.

\section{Altersforschung}

\section{Hundertjährige gesünder als vermutet}

- In Berlin, Brandenburg und Mecklenburg-Vorpommern waren 1.576 AOK Nordost-Versicherte im Jahr 2015 wenigstens 100 Jahre alt und nur 41,2\% davon lebten im Pflegeheim.

Das Gesundheitswissenschaftliche Institut der AOK Nordost (GeWINO) veröffentlichte jetzt Zahlen, wonach 23,2\% der 100+-Jährigen AOK Nordost-Versicherten 2015 ohne regelmäßig verschriebene Medikamente und einige sogar ganz ohne Verordnungen auskamen. Der Anteil der 100+-Jährigen, die mehr als fünf Wirkstoffgruppen verord- net bekamen, lag bei 34,3\%. „Erstaunlich ist, dass $35,5 \%$ der Hochbetagten nicht unter Demenz und fast drei Viertel der Studienpopulation nicht unter Depression litten", erläutert Prof. Dr.-Ing. Thomas P. Zahn, GeWINO. Im Analysejahr 2015 waren rund 65\% der Hochbetagten nicht im Krankenhaus. Fast alle Hochbetagten erhielten von 2009 bis 2015 mindestens eine Hilfsmittelverordnung. 31,7\% hatten wenigstens einen Arztkontakt pro Quartal.

\section{www.gewino.de}

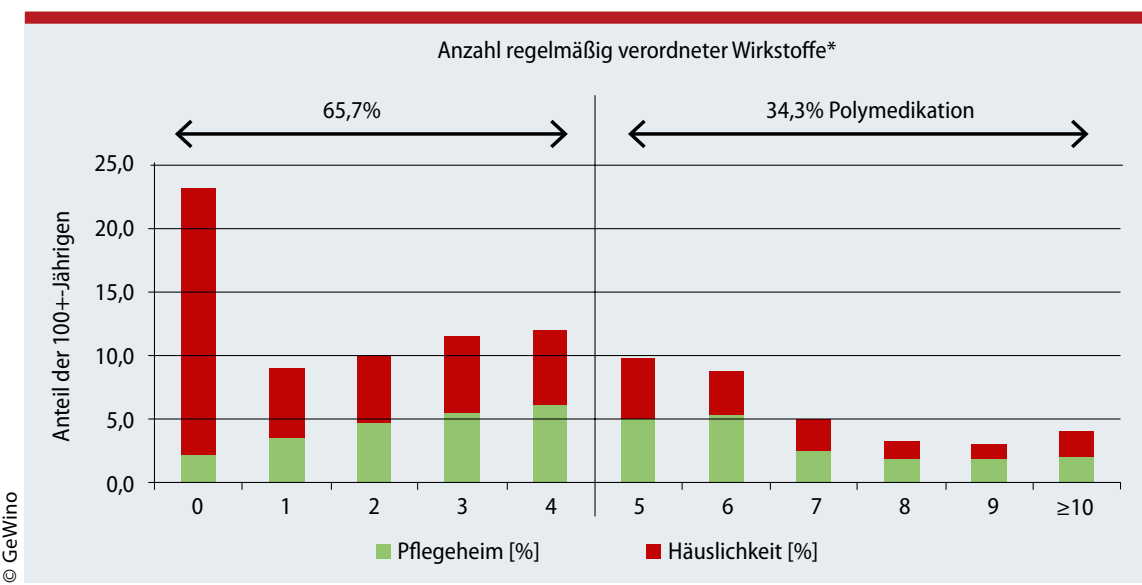

Abb. 1: Anzahl regelmäßig verordneter Wirkstoffe der 100+-Jährigen in 2015 [N = 1.576]

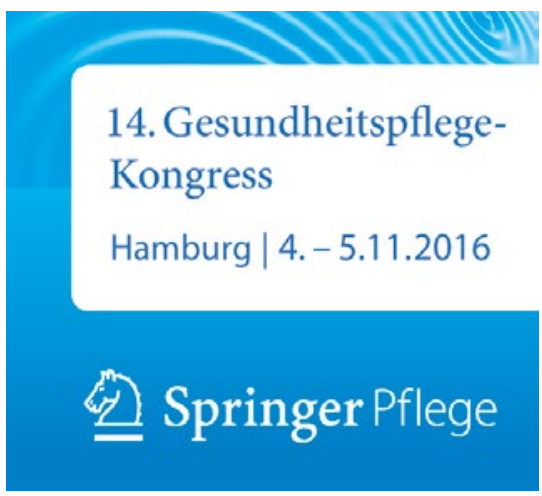

Ausblick: Am 20. und 21. Januar 2017 veranstaltet Springer Pflege im Berliner Hotel Maritim proArte den Kongress Pflege 2017. Neben Fortbildung ist er die Plattform für Networking und professionellen Austausch.

www.gesundheitskongresse.de
Pflegeausbildung

\section{DPR kritisiert}

\section{Hängepartie um Reform}

— Im Deutschen Bundestag liegt die Reform des Pflegeberufegesetzes auf Halde. „Wir fordern die Abgeordneten der Regierungskoalition auf, endlich die Beratungen zum Pflegeberufereformgesetz zu Ende zu führen und das Gesetz ohne faule Kompromisse zu beschließen", sagte Andreas Westerfellhaus, Präsident des Deutschen Pflegerats (DPR). Die seit Monaten andauernde Hängepartie um die künftige, moderne Pflegeausbildung verunsichere die gesamte Branche, schrecke Ausbildungsinteressierte ab und verstärke tagtäglich den Pflegefachpersonalmangel.

„Es geht bei der Reform ja nicht darum, den professionell Pflegenden einen Gefallen zu tun", so der DPR-Präsident. Es gehe schlicht darum, die Pflegeausbildung angesichts der enormen medizinischen und pflegerischen Herausforderungen zukunftstauglich zu machen. Westerfellhaus: „Geschieht das nicht, tragen vor allem kranke und pflegebedürftige Menschen den Schaden davon."

www.deutscher-pflegerat.de 